\title{
EXTRAÇÃO E CARACTERIZAÇÃO DO AMIDO DE DIFERENTES GENÓTIPOS DE BANANEIRA ${ }^{1}$
}

\author{
MAGALI LEONEL ${ }^{2}$, EZEQUIEL LOPES DO CARMO², SARITA LEONEL ${ }^{3}$, \\ CÉLIA MARIA LANDI FRANCO ${ }^{4}$, RAQUEL BOMBARDA CAMPANHA ${ }^{4}$
}

RESUMO- A banana, uma das principais herbáceas cultivadas em grande quantidade no mundo e importante fonte de energia para as pessoas, tem obtido interesse como matéria-prima para a produção de farinha e fécula para uso alimentício e outros fins industriais, devido ao elevado conteúdo de amido nos frutos verdes. O trabalho teve como objetivo caracterizar os frutos verdes de sete genótipos de bananeira quanto à composição química e avaliar o conteúdo de amilose, propriedades de pasta e térmicas dos amidos destes genótipos. Os resultados obtidos mostraram diferenças significativas na composição química dos frutos, com maior teor de amido na cultivar Figo Cinza. As análises dos amidos mostraram valores de amilose de 26,68 a 33,95\% e propriedades de pasta com elevado pico de viscosidade, baixa resistência à temperatura e agitação (quebra de viscosidade) e tendência à retrogradação para todos os amidos. Nas propriedades térmicas, os amidos mostraram um endoderma, temperatura de gelatinização na faixa de 60 a $73^{\circ} \mathrm{C}$ com DH variando de 11,6 a $16,99 \mathrm{~J}$ g-1 na gelatinização e 4,11 a 7,97 J g-1 na retrogradação.

Termos para indexação: Musa sp, frutos, propriedades térmicas, viscosidade.

\section{STARCH EXTRACTION AND CHARACTERIZATION OF DIFFERENT GENOTYPES OF BANANA}

\begin{abstract}
The banana, the largest herbaceous plant in the world, grown abundantly in many countries is considered to be one of the most important sources of energy for people. Due to high starch concentration, banana processing into flour and starch is of interest in view of a possibly important resource for food and other industrial purposes. The objective of this study was to characterize the chemical composition of green fruits of seven banana genotypes, and evaluate the content of amylose, pasting and thermal properties of starches. Results showed significant differences to chemical composition with higher level of starch in cultivar Figo cinza. The amylose content ranged from 26.68 to $33.95 \%$. Starches analysis showed pasting properties with high cold peak and breakdown, with retrogradation tendency to all genotypes. The thermal properties showed one endoderm. The gelatinization temperature was in range of 60 to $73^{\circ} \mathrm{C}$ with $\mathrm{DH}$ ranged from 11.6 to $16.99 \mathrm{~J} \mathrm{~g}-1$ in gelatinization and 4.11 to $7.97 \mathrm{~J} \mathrm{~g}-1$ in retrogradation.
\end{abstract}

Index terms: Musa sp, fruits, thermal properties, viscosity.

\section{INTRODUÇÃO}

A banana é uma das frutas mais consumidas no mundo, representando a quarta mercadoria alimentícia em volume de comercialização. Em 2007, a produção mundial atingiu 81,3 milhões de toneladas, destacando-se o Brasil, em 2009, como o terceiro país produtor, com uma produção de aproximadamente 7,1 milhões de toneladas (Agrianual, 2010).

Cultivada de norte a sul do Brasil, a banana tem grande expressão econômica e elevada impor- tância social como fonte barata de energia, minerais e vitaminas. Contudo, o País, um dos grandes produtores mundiais, apresenta como um dos principais problemas na comercialização o elevado desperdício pós-colheita, chegando, em algumas regiões, a 50\% da produção (Borges, 2003). Frente a isto, a banana verde vem sendo considerada um produto de grande interesse para a indústria alimentícia, devido às facilidades de transporte e armazenamento, bem como ao considerável teor de amido (MV, 2010).

Amidos de diferentes origens botânicas

\footnotetext{
${ }^{1}$ Trabalho Sinfruit 078 - Simpósio Internacional de Fruticultura - Avanços na Fruticultura (17 a 21 Outubro)

${ }^{2}$ UNESP.Centro de Raízes e Amidos Tropicais (CERAT), Caixa Postal 237, CEP 18610-307, Botucatu-SP. E-mail: mleonel@cerat.unesp.br ${ }^{3}$ UNESP. Faculdade de Ciências Agronômicas. Departamento de Produção Vegetal. Caixa Postal 237, CEP 18610-307, Botucatu-SP. E-mail: sarinel@fca.unesp.br

${ }^{4}$ UNESP. Instituto de Biociências, Letras e Ciências Exatas. Departamento de Engenharia e Tecnologia de Alimentos. Rua Cristovão Colombo, 2265, CEP 15054-000, São José do Rio Preto-SP. E-mail:celia@ibilce.unesp.br
} 
possuem diversas propriedades funcionais, o que os torna úteis para aplicações em alimentos, produtos farmacêuticos, papel, plástico e outros setores industriais (NWOKOCHA et al., 2009).

O amido é uma matéria-prima abundante, renovável, biodegradável e não tóxica que pode ser extraído com elevada pureza por meio de processos industriais relativamente simples e ser facilmente convertido em várias substâncias por processos químicos e bioquímicos. Esses fatores, somados, determinam o imenso potencial agrícola e industrial do amido.

O amido é um polissacarídeo constituído apenas de resíduos de glicose, sendo assim considerado um homopolissacarídeo. Os dois principais componentes do amido, amilose e amilopectina, são formados por resíduos de D-glicose unidos por ligações $\alpha-(1,4)$ e ligações $\alpha-(1,6)$ nos pontos de ramificação (BULÉON et al., 1998). A amilopectina é uma molécula altamente ramificada, enquanto a amilose apresenta poucas ramificações (JANE, 2006). A proporção entre amilose e amilopectina é variável com a fonte botânica, o que irá conferir características específicas à pasta de amido, assim como à organização física das mesmas dentro da estrutura granular (BILIADERIS, 1991).

A disposição de amilose e amilopectina dentro do grânulo de amido ainda não é completamente compreendida, porém o empacotamento desses dois polímeros no grânulo de amido nativo é bem organizado. No entanto, quando aquecido na presença de água, a estrutura do grânulo torna-se menos ordenada, e esta perda na organização interna ocorre em diferentes temperaturas para vários tipos de amidos. O conteúdo desses polissacarídeos afeta a arquitetura do grânulo de amido, as propriedades de pasta e os atributos texturais, podendo afetar sua aplicação em alimentos industrializados (THOMAS; ATWELL, 1999).

A principal propriedade do amido é a gelatinização, capacidade de tornar-se viscoso em água com o aumento da temperatura. As propriedades de pasta e térmicas de amidos podem ajudar a determinar sua funcionalidade específica e, portanto, sua utilidade na indústria de alimentos (KRIEGER et al., 1997).

Diante da importância da cultura da banana no Brasil e da possibilidade de utilização da banana-verde como fonte de amido, este trabalho objetivou caracterizar frutos verdes de diferentes genótipos de bananeira quanto à composição química, extrair e analisar seus amidos para o teor de amilose, propriedades de pasta e térmicas, visando a fornecer informações importantes para o setor.

\section{MATERIAL E MÉTODOS}

\section{Análises dos frutos}

Nove genótipos de bananeira procedentes do pomar experimental do Departamento de Produção Vegetal - Horticultura, da Faculdade de Ciências Agronômicas - FCA/UNESP, foram selecionados: 'Caipira', 'Figo Cinza', 'Fhia 01', 'Nam', 'Nanicão IAC', 'Prata-Anã' e seu híbrido 'FHIA-18'.

Os frutos utilizados foram colhidos do cacho inteiro, no estádio 1 de maturação, de acordo com Von Loesecke (FAEP, 2009) (casca totalmente verde), descartando-se aqueles que estavam fora do padrão, higienizados com água potável e posteriormente descascados. Em seguida, foram fatiados em espessuras de aproximadamente $0,6 \mathrm{~cm}$, retirada uma amostragem para análise de umidade (AACC, 1983). O restante foi acondicionado em bandejas e levados para estufa com circulação de ar forçado, a $50^{\circ} \mathrm{C}$. Após 48 horas, as fatias desidratadas foram retiradas e moídas em moinho tipo faca. As análises foram realizadas em três repetições, utilizando-se o material desidratado dos respectivos genótipos. No material desidratado, foram realizadas as análises dos teores de: umidade, cinzas, matéria graxa, fibra bruta, proteína, açúcares redutores, açúcares solúveis totais e amido (AACC, 1983; Nelson, 1944; Somogy, 1945).

\section{Extração dos amidos}

Os frutos verdes foram descascados e homogeneizados com solução gelada $\left(4^{\circ} \mathrm{C}\right)$ de ácido ascórbico $(1 \%)$ na proporção de $0,25 \mathrm{~kg} /$ litro em liquidificador industrial por 2 minutos. Em seguida, o material foi passado em peneira de 60 Tyler e deixou-se decantar por 4 horas a $5^{\circ} \mathrm{C}$. Decorrido este tempo, descartou-se o líquido sobrenadante, e o amido decantado foi ressuspenso em água, passou por peneira de 200 Tyler e foi colocado para decantar por 12 horas a $5^{\circ} \mathrm{C}$. O líquido sobrenadante foi novamente descartado, e o amido obtido foi ressuspenso em água e centrifugado a $3.000 \mathrm{rpm}$ por 15 minutos, descartando-se o sobrenadante, e sendo seco em estufa com circulação de ar a $40^{\circ} \mathrm{C}$. Após a secagem, as amostras passaram por moinho de facas, e os amidos foram armazenados em frascos de vidro à temperatura ambiente.

\section{Análises dos amidos}

Para a análise do teor de amilose, os amidos extraídos dos frutos verdes dos diferentes genótipos de bananeira foram desengordurados, conforme 
descrito por Franco et al. (2002). Os teores de amilose foram determinados segundo a metodologia de Williams et al. (1970).

As propriedades de pasta foram analisadas através do Rapid Visco Analyser (RVA). As amostras foram pesadas de acordo com as respectivas umidades e acrescentados aproximadamente $25 \mathrm{ml}$ de água e avaliadas pelo programa de tempo e temperatura 2Std2 do software Thermocline for Windows, versão 2.2. Foram avaliadas a temperatura de pasta, a viscosidade máxima (pico), a queda de viscosidade ou "breakdown" (diferença entre a viscosidade máxima e a viscosidade da pasta mantida a $95^{\circ} \mathrm{C}$ por 5 minutos), a viscosidade final e a tendência à retrogradação (diferença entre a viscosidade final e a viscosidade da pasta a $50^{\circ} \mathrm{C}$ por 5 minutos). Os resultados foram expressos em RVU (1 RVU= aprox. 12cP) (NEWPORT SCIENTIFIC, 1998).

As propriedades térmicas de gelatinização e retrogradação foram analisadas através do Calorímetro Diferencial de Varredura (DSC) Pyris 1 - (Perkin Elmer, USA). Foram pesados $4 \mathrm{mg}$ (base seca) dos respectivos amidos em recipientes apropriados de alumínio, adicionados $6 \mu \mathrm{L}$ de água deionizada e posteriormente seladas. As amostras foram mantidas por 2 horas em temperatura ambiente para equilíbrio e, em seguida, colocadas no calorímetro e aquecidas a $5^{\circ} \mathrm{C}$ por minuto, de 25 até $100^{\circ} \mathrm{C}$, além da utilização de um recipiente apropriado de alumínio vazio utilizado como referência. Após a varredura nas amostras, estas foram armazenadas em temperatura de refrigeração por 15 dias. Sequencialmente, foram avaliadas as propriedades térmicas dos amidos retrogradados, seguindo as mesmas condições de análise para gelatinização. As temperaturas inicial, de pico, final e a variação de entalpia dos amidos foram determinadas através do software Pyris $1 \mathrm{da}$ Perkin Elmer (USA).

\section{RESULTADOS E DISCUSSÃO}

\section{Composição química dos frutos}

As análises da composição química dos frutos verdes dos diferentes genótipos de bananeira (Tabela 1) mostram que estes diferiram quanto aos componentes, com os teores de umidade nos frutos verdes variando de $59,7 \%$ a 77,20\%, sendo o 'Figo Cinza' o que apresentou o menor teor de umidade nos frutos e, consequentemente, maior teor de matéria seca. Para os teores de cinzas, os valores variaram de 0,94 a $1,51 \%$, os de matéria graxa de 0,09 a $0,31 \%$, proteínas de 1,07 a 1,54\%, açúcares redutores de 0,10 a $0,45 \%$ e açúcares totais de 0,28 a $1,51 \%$. Os frutos verdes da cultivar Nam apresentaram os maiores teores de cinzas, matéria graxa, proteína, açúcares redutores e totais.

Já para os teores de fibras $(0,33$ a $1,14 \%)$ e amido (14,71 a 27,2\%), os frutos de 'Figo Cinza' apresentaram os maiores valores. Ramos e Leonel (2009), avaliando a qualidade dos frutos de bananeiras de diferentes genótipos, verificaram teores de amido que variaram de 12,04 a 16,07\% nos mesmos genótipos avaliados neste trabalho. Os menores valores observados podem ser devidos ao estádio de maturação dos frutos.

\section{Análises dos amidos}

Os resultados obtidos para os teores de amilose diferiram entre os genótipos de bananeira, sendo que os maiores teores foram observados em 'Nam' e 'Figo cinza', não ocorrendo diferença significativa entre os demais amidos (Tabela 2).

O conteúdo de amilose no amido de banana tem sido reportado em diversos trabalhos com grandes variações. Ling et al. (1982) e Garcia e Lajolo (1988) encontraram 19,5\% e 17\%, respectivamente, em banana Cavendish, enquanto Enggleston et al. (1992), estudando amidos extraídos de bananas nativas e híbridas, observaram variação de 9 a 12\% de amilose. Waliszewski et al. (2003) reportaram $40,7 \%$, e Nwokocha e Willians (2009) encontraram valores de $26 \%$ de amilose aparente e $24 \%$ de amilose total para amido de variedades branca e amarela de banana.

O perfil de viscosidade é extremamente útil na determinação do comportamento do amido sob várias condições e para comparar diferenças relativas entre amidos. Os resultados obtidos para as propriedades de pasta dos amidos de banana estão apresentados na Tabela 2.

Quando suspensões aquosas de amido são submetidas ao RVA, há aumento da viscosidade na fase inicial de aquecimento, período em que os grânulos também iniciam o poder de inchamento, e moléculas de amilose são lixiviadas desses amidos (FERNÁNDEZ-MUÑOZ et al., 2011). Um pico de viscosidade é obtido durante o empastamento, quando existe a maioria dos grânulos totalmente inchados, grânulos intactos, e o alinhamento molecular de qualquer polímero solubilizado ainda não ocorreu dentro do campo de atrito do instrumento. Durante a fase de temperatura constante $\left(95^{\circ} \mathrm{C}\right)$, os grânulos começam a se quebrar, e a solubilização dos polímeros continua. Neste ponto, ocorre quebra na viscosidade. Durante a fase de resfriamento, polímeros de amilose e amilopectina solubilizados começam a se reassociar, 
e outro aumento na viscosidade é registrado. Este segundo aumento da viscosidade é conhecido como tendência a retrogradação.

Os resultados obtidos evidenciaram diferenças significativas entre os genótipos de bananeira para as propriedades de pasta de seus amidos. O amido da bananeira 'Caipira' apresentou o maior pico de viscosidade, e o menor pico foi observado no amido da cultivar Nam. Já com relação á quebra de viscosidade, que indica a resistência à temperatura e agitação, 'Nanicão IAC 2001' apresentou amido com maior resistência, ou seja, menor quebra de viscosidade. Todos os amidos apresentaram aumento da viscosidade com o resfriamento e tendência à retrogradação, com o maior valor observado no amido de 'Figo cinza'.

As mudanças que ocorrem nos grânulos de amido durante a gelatinização e retrogradação são os principais determinantes do comportamento de pasta desses amidos. Os resultados da análise térmica de gelatinização e retrogradação dos amidos de banana mostraram diferenças entre os genótipos (Tabela 3 ).

Cada amido tem suas temperaturas características de gelatinização, Ti, Tp e Tf (temperaturas inicial, de pico e final, respectivamente) e sua entalpia de gelatinização $(\Delta \mathrm{H})$ obtidas do DSC (TESTER, 1997). Depois de atingida a temperatura final (Tf), as duplas hélices da amilopectina são dissociadas, embora a expansão da estrutura granular possa ser conservada até que temperaturas mais altas e cisalhamentos sejam aplicados. Acima de $95^{\circ} \mathrm{C}$, um gel amorfo é formado. O intervalo de temperatura Tf-Ti representa o período de gelatinização (BLENNOW et al., 2000).

De acordo com os resultados obtidos pelo DSC, apenas um endoderma foi obtido para todos os amidos de banana, ou seja, não há amilose complexada com lipídeos, o que levaria a um segundo endoderma (YOO; Jane, 2002). Os amidos de 'FHIA 18 ' e 'Nanicão IAC' apresentaram as mais altas temperaturas de gelatinização, o que pode indicar cadeias longas ramificadas de amilopectina. Por outro lado, 'Figo cinza' e 'FHIA 01' apresentaram as menores temperaturas de gelatinização.

Os amidos de 'FHIA 18', 'Prata-Anã' e 'Caipira' apresentaram elevada entalpia de gelatinização (DH), o que pode estar relacionado com a cristalinidade. As propriedades de gelatinização e de inchamento são controladas, em parte, pela estrutura da amilopectina. Como as regiões cristalinas do grânulo de amido são geralmente compostas de amilopectina, amidos com alto teor de amilose apresentam baixas temperaturas de gelatinização (inicial e de pico) e entalpia (NODA et al., 1998).

A cristalinidade do amido pode ser estimada pela entalpia $(\Delta \mathrm{H})$, energia necessária para fundir os segmentos de duplas hélices. As temperaturas de gelatinização (inicial e de pico) podem ser usadas como medida da estabilidade ou perfeição das regiões cristalinas e a largura do pico endotérmico, como estimativa da heterogeneidade da estrutura cristalina (BLENNOW et al., 2000).

O comportamento de amidos gelatinizados, quando armazenados e resfriados, geralmente expresso como retrogradação, é de grande interesse para cientistas e tecnólogos na área de alimentos, pois afeta profundamente a qualidade, aceitabilidade e meia-vida de muitos alimentos baseados em amido (KARIM; NORZIAH; SEOW, 2000).

$\mathrm{Na}$ avaliação das propriedades térmicas de retrogradação de amidos de banana, todos os valores dos parâmetros Ti, Tp, Tf e $\Delta \mathrm{H}$ foram inferiores aos apresentados nas propriedades turmicas de gelatinização. A retrogradação é o processo pelo qual o amido retorna ao seu estado mais ordenado, após a gelatinização (KLUCINEC; THOMPSON, 1999). Durante a retrogradação, as moléculas de amilose formam associações de duplas hélices de 40 a 70 unidades de glicose, sendo que a amilopectina forma duplas hélices menores, devido às restrições impostas pela estrutura ramificada das moléculas e o comprimento das ramificações. Essa reassociação ocorre de forma molecular e estrutural mais fraca que a encontrada na molécula nativa. Portanto, menos energia é requerida para fundir os cristais reestruturados (SASAKI; YASUI; MATSUKI, 2000). 
TABELA 1- Composição química de frutos verdes de diferentes genótipos de bananeira.

\begin{tabular}{lcccccccc}
\hline Genótipos & Umidade & Cinzas & $\begin{array}{c}\text { Matéria } \\
\text { Graxa }\end{array}$ & Fibra & Proteína & $\begin{array}{c}\text { Açúcares } \\
\text { redutores }\end{array}$ & $\begin{array}{c}\text { Açúcares } \\
\text { totais }\end{array}$ & Amido \\
\hline Prata-Anã & $67,9 \mathrm{~b}$ & $0,94 \mathrm{e}$ & $0,20 \mathrm{~b}$ & $0,48 \mathrm{c}$ & $1,12 \mathrm{e}$ & $0,20 \mathrm{e}$ & $0,46 \mathrm{e}$ & $20,9 \mathrm{bc}$ \\
\hline Fhia 18 & $75,91 \mathrm{a}$ & $1,16 \mathrm{bc}$ & $0,14 \mathrm{~cd}$ & $0,70 \mathrm{~b}$ & $1,07 \mathrm{f}$ & $0,40 \mathrm{ab}$ & $0,48 \mathrm{e}$ & $16,7 \mathrm{~d}$ \\
\hline Fhia 01 & $72,6 \mathrm{ab}$ & $0,95 \mathrm{e}$ & $0,17 \mathrm{bc}$ & $0,36 \mathrm{c}$ & $1,26 \mathrm{~cd}$ & $0,38 \mathrm{~b}$ & $0,70 \mathrm{~d}$ & $19,8 \mathrm{c}$ \\
\hline Nanicão IAC & $73,8 \mathrm{ab}$ & $1,02 \mathrm{de}$ & $0,17 \mathrm{bc}$ & $0,77 \mathrm{~b}$ & $1,23 \mathrm{c}$ & $0,32 \mathrm{c}$ & $1,20 \mathrm{~b}$ & $20,3 \mathrm{c}$ \\
\hline Caipira & $77,20 \mathrm{a}$ & $1,24 \mathrm{~b}$ & $0,08 \mathrm{ef}$ & $0,33 \mathrm{c}$ & $1,31 \mathrm{~b}$ & $0,26 \mathrm{de}$ & $0,91 \mathrm{c}$ & $14,71 \mathrm{e}$ \\
\hline Nam & $71,8 \mathrm{ab}$ & $1,51 \mathrm{a}$ & $0,31 \mathrm{a}$ & $0,40 \mathrm{c}$ & $1,54 \mathrm{a}$ & $0,45 \mathrm{a}$ & $1,51 \mathrm{a}$ & $20,8 \mathrm{c}$ \\
\hline Figo Cinza & $59,7 \mathrm{c}$ & $1,12 \mathrm{~cd}$ & $0,09 \mathrm{def}$ & $1,14 \mathrm{a}$ & $1,31 \mathrm{~b}$ & $0,10 \mathrm{f}$ & $0,28 \mathrm{f}$ & $27,2^{\mathrm{a}}$ \\
\hline C.V. & 3,07 & 3,28 & 12,51 & 10,33 & 1,16 & 4,84 & 6,16 & 2,79 \\
\hline
\end{tabular}

Médias seguidas de mesma letra na coluna não diferem a 5\%, pelo teste de Tukey.

TABELA 2- Teor de amilose e propriedades de pasta dos amidos de banana.

\begin{tabular}{lccccc}
\hline Genótipos & Amilose (\%) & \multicolumn{4}{c}{ Propriedades de pasta (RVU) } \\
\cline { 3 - 6 } & & $\begin{array}{c}\text { Pico de } \\
\text { viscosidade }\end{array}$ & $\begin{array}{c}\text { Quebra de } \\
\text { viscosidade }\end{array}$ & Viscosidade final & $\begin{array}{c}\text { Tendência à } \\
\text { retrogradação }\end{array}$ \\
Prata-Anã & $29,55 \mathrm{~b}$ & $303,80 \mathrm{~d}$ & $141,25 \mathrm{~d}$ & $241,67 \mathrm{f}$ & $79,13 \mathrm{~d}$ \\
Fhia 18 & $29,53 \mathrm{~b}$ & $300,21 \mathrm{~d}$ & $74,04 \mathrm{f}$ & $308,67 \mathrm{~b}$ & $82,50 \mathrm{~d}$ \\
Fhia 01 & $28,96 \mathrm{~b}$ & $411,42 \mathrm{~b}$ & $215,55 \mathrm{~b}$ & $258,50 \mathrm{e}$ & $62,63 \mathrm{e}$ \\
Nanicão IAC & $28,00 \mathrm{~b}$ & $356,59 \mathrm{c}$ & $98,88 \mathrm{e}$ & $347,84 \mathrm{a}$ & $90,13 \mathrm{c}$ \\
Caipira & $26,68 \mathrm{~b}$ & $460,59 \mathrm{a}$ & $257,25 \mathrm{a}$ & $268,21 \mathrm{~d}$ & $64,88 \mathrm{e}$ \\
Nam & $32,07 \mathrm{a}$ & $185,00 \mathrm{e}$ & $37,17 \mathrm{~g}$ & $285,08 \mathrm{c}$ & $137,25 \mathrm{~b}$ \\
Figo Cinza & $33,95 \mathrm{a}$ & $302,75 \mathrm{~d}$ & $164,75 \mathrm{c}$ & $350,42 \mathrm{a}$ & $212,42 \mathrm{a}$ \\
\hline
\end{tabular}

Médias seguidas de mesma letra na coluna não diferem a 5\%, pelo teste de Tukey.

TABELA 3- Propriedades térmicas dos amidos de banana.

\begin{tabular}{|c|c|c|c|c|c|}
\hline \multirow[b]{2}{*}{ Genótipos } & \multicolumn{5}{|c|}{ Propriedades térmicas de gelatinização } \\
\hline & $\begin{array}{c}\text { T inicial } \\
\left({ }^{\circ} \mathrm{C}\right)\end{array}$ & $\begin{array}{c}\text { T pico } \\
\left({ }^{\circ} \mathrm{C}\right)\end{array}$ & $\begin{array}{l}\text { T final } \\
\left({ }^{\circ} \mathrm{C}\right)\end{array}$ & $\Delta \mathrm{T}(\mathrm{tf}-\mathrm{ti})$ & $\mathrm{DH}\left(\mathrm{J} \mathrm{g}^{-1}\right)$ \\
\hline Prata-Anã & $63,29 \mathrm{~b}$ & $66,68 \mathrm{bc}$ & $69,96 \mathrm{bc}$ & $6,68 \mathrm{a}$ & $14,95 \mathrm{ab}$ \\
\hline Fhia 18 & $66,98 \mathrm{a}$ & $69,89 \mathrm{a}$ & $73,35 \mathrm{a}$ & $6,38 \mathrm{a}$ & $16,99 \mathrm{a}$ \\
\hline Fhia 01 & $61,40 \mathrm{c}$ & $65,16 \mathrm{~cd}$ & $68,29 \mathrm{c}$ & $6,89 \mathrm{a}$ & $14,52 \mathrm{bc}$ \\
\hline Nanicão IAC & $66,28 \mathrm{a}$ & $69,51 \mathrm{a}$ & $73,23 \mathrm{a}$ & $6,96 \mathrm{a}$ & $12,44 \mathrm{~cd}$ \\
\hline Caipira & $63,81 \mathrm{~b}$ & $66,96 \mathrm{~b}$ & $70,00 \mathrm{~b}$ & $6,19 \mathrm{a}$ & $15,01 \mathrm{ab}$ \\
\hline Nam & $63,19 \mathrm{~b}$ & $66,08 \mathrm{bc}$ & $69,79 \mathrm{bc}$ & $6,60 \mathrm{a}$ & $12,63 \mathrm{bcd}$ \\
\hline Figo Cinza & $60,93 \mathrm{c}$ & $64,37 \mathrm{~d}$ & $68,25 \mathrm{c}$ & $7,32 \mathrm{a}$ & $11,60 \mathrm{~d}$ \\
\hline \multirow[t]{3}{*}{ CV $(\%)$} & 0,60 & 0,57 & 0,38 & 4,30 & 3,94 \\
\hline & \multicolumn{5}{|c|}{ Propriedades térmicas de retrogradação } \\
\hline & $\begin{array}{c}\text { T inicial } \\
\left({ }^{\circ} \mathrm{C}\right)\end{array}$ & $\begin{array}{l}\text { T pico } \\
\left({ }^{\circ} \mathrm{C}\right)\end{array}$ & $\begin{array}{l}\text { T final } \\
\left({ }^{\circ} \mathrm{C}\right)\end{array}$ & $\Delta \mathrm{T}(\mathrm{tf}-\mathrm{ti})$ & $\mathrm{DH}\left(\mathrm{J} \mathrm{g}^{-1}\right)$ \\
\hline Prata-Anã & $37,58 \mathrm{~d}$ & $50,25 \mathrm{bc}$ & $62,90 \mathrm{~d}$ & $25,32 \mathrm{ab}$ & $6,16 c$ \\
\hline Fhia 18 & $37,63 d$ & $51,57 \mathrm{a}$ & $64,69 a$ & $27,06 a$ & $7,97 \mathrm{a}$ \\
\hline Fhia 01 & $39,87 \mathrm{ab}$ & $50,07 \mathrm{c}$ & $60,98 \mathrm{e}$ & $21,11 \mathrm{~b}$ & $4,11 \mathrm{e}$ \\
\hline Nanicão IAC & $39,17 \mathrm{c}$ & $51,90 \mathrm{a}$ & $64,15 b$ & $24,98 \mathrm{ab}$ & $6,24 c$ \\
\hline Caipira & $39,64 b c$ & $51,85 \mathrm{a}$ & $63,58 \mathrm{c}$ & $23,94 b$ & $6,96 b$ \\
\hline Nam & $39,69 b c$ & $50,52 b$ & $63,34 c$ & $23,65 b$ & $5,71 d$ \\
\hline Figo Cinza & $40,31 \mathrm{a}$ & $51,90 \mathrm{a}$ & $64,55 \mathrm{ab}$ & $24,24 \mathrm{~b}$ & $6,30 \mathrm{c}$ \\
\hline $\mathrm{CV}(\%)$ & 0,55 & 0,31 & 0,24 & 8,79 & 1,67 \\
\hline
\end{tabular}

Médias seguidas de mesma letra na coluna não diferem a 5\%, pelo teste de Tukey. 


\section{CONCLUSÕES}

1. Os frutos verdes dos genótipos de bananeira apresentam elevada umidade, sendo o amido o principal componente da matéria seca. O genótipo 'Figo cinza' destaca-se pela baixa umidade e elevado teor de amido.

2. Os amidos extraídos apresentam consideráveis teores de amilose (26,68 a 33,95\%), elevados picos de viscosidade e viscosidade final, tendência à retrogradação e faixa de gelatinização de 60 a $73^{\circ} \mathrm{C}$.

\section{REFERÊNCIAS}

AMERICAN ASSOCIATION OF CEREAL CHEMISTS. Approved methods of the American Association of Cereal Chemists. Saint Paul, 1983. v.1. AGRIANUAL 2010: anuário da agricultura brasileira. São Paulo: AgraFNP, 2010. 520p.

BILIADERIS, C. G. The structure and interactions of starch with food contituints. Canadian Journal Physiology Pharmacology, Birmingham, v. 69, n. 1, p. 60-78, 1991.

BORGES, M. T. M. R. Potencial vitamínico de banana-verde e produtos derivados. 2003. $60 \mathrm{f}$. Tese (Doutorado em Ciência de Alimentos) - Faculdade de Engenharia de Alimentos, Universidade Estadual de Campinas, Campinas, 2003.

BLENNOW, A. et al. The distribution of covalently bound phosphate in the starch granule in relation to starch crystallinity. International Journal of Biological Macromolecules, Maryland Heights, v. 27, n. 3, p. 211-218, 2000

BULÉON, A. et al. Starch granules: structure and biosynthesis - Mini review. Internacional Journal of Biological Macromolecules, Maryland Heights, v. 23, n. 2, p. 85-112, 1998.

EGGLESTON, G.; SWENNEN, R.; AKONI, S. Physicochemical studies on starches isolated from plantain cultivars, plantain hybrids and cooking bananas. Starch/Stärke, Weinheim, v. 44, p. 121$128,1992$.

FEDERAÇÃO DA AGRICULTURA DO ESTADO DO PARANÁ. Cartilhas de classificação. Disponível em: $<$ http://www.faep.com.br $>$. Acesso em: 25 fev. 2009.
FERNÁNDEZ-MUÑOZ, J. L. et al. Effect of calcium content in the corn flour on RVA profiles. Journal of Food Engineering, London, v. 102, n. 1, p. 100103, 2011.

FRANCO, C. M. L.; WONG, K.S.; YOO, S.H.; JANE, J.L. Structural and functional characteristics of selected soft wheat starches. Cereal Chemistry, Saint Paul, v. 79, n. 2, p. 243248, 2002.

GARCIA, E.; LAJOLO, F. M. Starch transformation during banana ripening: the amylase and glucosidase behavior. Journal of Food Science, Chicago, v. 53 , n. 4, p. 1181-1186, 1988.

JANE, J. Current understanding on starch granule structure. Journal of Applied Glycoscience, Tokyo, v. 53, n. 3, p. 205-213, 2006.

KARIM, A.; NORZIAH, M. H.; SEOW, C. C. Methods for the study of starch retrogradation. Food Chemistry, Whiteknights, v. 71, n. 1, p. 9-36, 2000.

KLUCINEC, J. D.; THOMPSON, D. B. Amylose and amylopectin interact in retrogradation of dispersed high-amylose starches. Cereal Chemistry, Saint Paul, v. 76, n. 2, p. 282-291, 1999.

KRIEGER, K. M. et al. Thermal properties of corn starch extracted with different blending methods: micro blender and homogeneizer. Cereal Chemistry, Saint Paul, v. 74, n. 5, p. 553-555, 1997.

Ling, L. H. et al. Physical properties of starch from Cavendish banana fruit. Starch/Stärke, Weinheim, v. 34, 184-188, 1982.

MV - Minha Vida. Os poderes da banana verde. Disponível em: <http://www.minhavida.com.br $>$. Acesso em: 29 jun. 2010.

NELSON, N. A. Photometric adaptation of the Somogy method for the determination of glucose. Journal Biology Chemistry, Baltimore, n. 153, p. 375-380, 1944.

NEWPORT SCIENTIFIC. Operation manual for series 4: instructions manual. Warriedwood, 1998. $42 \mathrm{p}$ 
NODA, T.; TAKAHATA, Y.; SATO, T.; SUDA, I.; MORISHITA, T.; ISHIGURO, K.; YAMAKAWA, O. Relationships between chain lenght distribution of amylopectin and gelatinization properties within the same botanical origin for sweet potato and buckwheat. Carbohydrate Polymers, Barking, v. 37, n. 2, p. 153-158, 1998.

NWOKOCHA, L. M.; WILLIANS, P. A. Some properties of white and yellow plantain (Musa paradisiaca, Normalis) starches. Carbohydrate Polymers, Barking, v. 76, p. 133-138, 2009.

NWOKOCHA, L. M.; AVIARA, N.A.; SENAN, C.; WILLIAMS, P.A. A comparative studie of some properties of cassava (Manihot esculenta, Crantz) and cocoyam (Colocasia esculenta, Linn) starches. Carbohydrate Polymers, Barking, v. 76, p. 362367, 2009.

RAMOS, D. P.; LEONEL, S.; MISCHAN, M. M. Caracterização físico-química dos frutos de genótipos de bananeira produzidos em Botucatu-SP. Ciência e Agrotecnologia, Lavras, v. 33, p. 1765-1770, 2009.

SASAKI, T.; YASUI, T.; MATSUKI, J. Effect of amylose content on gelatinization, retrogradation, and pasting properties of starch from waxy and nonwaxy wheat and their F1 seeds. Cereal Chemistry, Saint Paul, v. 77, n. 1, p. 58-63, 2000.
SOMOGY, M. Determination of blood sugar. Journal Biology Chemistry, Baltimore, n. 160, p. 69-73, 1945.

TESTER, R. F.; KARKALAS, J.; QI, X. Starch structure and digestibility enzyme-substrate relationship. World's Poultry Science Journal, Cambridge, v. 60, n. 2, p. 186-195, 2004.

THOMAS, D. J.; ATWELL, W. A. Starches: practical guides for the food industry. Minnesota: Eagan Press, 1999. $94 \mathrm{p}$.

WALISZEWSKI, K. N.; APARICIO, M.A.; BELLO, L.A.; MONROY, J.A. Changes of banana starch by chemical and physical modification. Carbohydrate Polymers, Barking, v. 52, p. 237-242, 2003.

WILLIAMS, P.C.; KUZINA, F.D.; HLYNKA, I. A rapid colorimetric method for estimating the amylose content of starches and flours. Cereal Chemistry, Saint Paul, v.47, n.4, 1970.

YOO, S,H.; JANE, J,I. Structural and physical characteristics of waxy and other wheat starches. Carbohydrate Polymers, Barking, v. 49, p. 297-305, 2002 